\title{
10.2478/v10170-011-0024-0
}

Archives of Control Sciences

Volume 22(LVIII), 2012

No. 3, pages $273-284$

\section{A control Lyapunov function approach to adaptive control of HIV-1 infection}

\author{
JOÃO M. LEMOS and MIGUEL S. BARÃO
}

\begin{abstract}
This paper presents an algorithm for nonlinear adaptive control of the viral load in HIV-1 infection. The infection model considered is a reduced complexity nonlinear state-space model with two state variables, that represent the plasma concentration of uninfected and infected CD4+ T-cells of the human immune system. The viral load is assumed to be proportional to the concentration of infected cells. First, a change of variables that exactly linearizes the system is obtained. For the resulting linear system the manipulated variable is obtained by state feedback. To compensate for the uncertainty in the infection parameter of the model an estimator based on a Control Lyapunov Function is designed.
\end{abstract}

Key words: nonlinear adaptive control, HIV-1 infection, immunology, exact linearization, control Lyapunov function

\section{Introduction}

\subsection{Framework}

Strategies for counteracting HIV infection designed using control methods are receiving an increased attention. Detailed studies that combine modeling analysis with clinical results show that the initial infection phase may be represented using simple nonlinear state models [6]. This fact boosted the production of an increasing number of papers where therapy strategies are derived from control principles.

A straightforward approach to the design of a controller to regulate the state of a nonlinear system consists in obtaining an approximate linear model around the equilibrium point considered using Taylor series approximations and then to design a state feedback controller that drives the state increments with respect to the equilibrium to zero. Although simple, this method has the drawback of requiring that the initial conditions are close to the equilibrium for the approximation to be valid, being difficult to establish

The Authors are with INESC-ID/IST and INESC-ID/Univ. Évora, R. Alves Redol 9, 1000-029 Lisboa, Portugal, $\{$ jlml,jmsb $\} @$ inesc-id.pt

This work was performed in the framework of project HIVCONTROL - Control based on dynamic modeling of HIV-1 infection for therapy design, supported by Fundação de Ciência e Tecnologia, Portugal, under contract PTDC/EEA-CRO/100128/2008.

Received 30.04.2012. 
stability results. Furthermore, if the linearized system is not controllable, it may not be possible to design adequately the state feedback. This is the case of the model of HIV-1 infection considered hereafter around the equilibrium corresponding to an healthy person. If this approach is followed, the linearization must then be performed around the equilibrium point corresponding to infection and the state feedback controller should thus drive the state away from it, with the risk of becoming unstable due to the neglected higher order terms of the model.

Opposite to this approach, feedback linearization [5] aims at exactly canceling the nonlinearities using a nonlinear static feedback. This results in a transformed model that is exactly linear in a region around the equilibrium point to which a linear regulator may then be applied. In this region, that is usually larger than the one resulting from Taylor approximation methods, stability of the closed loop is ensured.

This paper proposes a strategy that combines model reduction using a simple singular perturbation approximation, feedback linearization and LQ regulation based on state feedback. Due to the wide variability of the dynamics associated to different patients the capacity of a controller to stabilize models that are different from the nominal one is quite important. Hence, we consider the inclusion of an estimator of the infection parameter.

It should be remarked that the present paper, as well as the references quoted above, forms just one step towards the application of control techniques to the design of HIV1 infection therapy. Indeed, in the actual clinical practice, the drugs currently used for treatment of HIV-1 infection are neither continuously infused nor is the virus concentration measured in permanence. The sampling of the controllers designed is therefore required, a subject that deserves attention on its own from the point of view of systems and control.

\subsection{Literature review}

Examples of research papers addressing the design of HIV-1 infection therapy with control techniques include nonlinear control based on Lyapunov methods and on the use of decomposition in strict feedback form with backstepping [4], adaptive control [3], Optimal Control [8] and Predictive Control [10]. In [2] various methods based on timedelay feedback control are shown, via Lyapunov function methods, to stabilize an HIV1 model similar to the one considered in the present paper. In [1] a HIV-1 infection control strategy based on nonlinear geometric control (exact linearization) is described, but without any mention to adaptive control and considering a different model.

\subsection{Paper contributions and organization}

The contribution of this paper consists of a therapy design procedure for HIV-1 based on nonlinear adaptive control. The controller proposed combines exact linearization with an adaptation mechanism that relies on a joint control Lyapunov function for the tracking and estimation errors. 
The paper is organized as follows: After this introduction that motivates the problem, reviews the main references and states the paper contribution and structure, the model used for HIV-1 infection is presented in section 2. This model has two equilibrium points that are characterized in section 3. Section 4 addresses exact linearization and section 5 describes the design of the controller for the resulting linear system. The main contribution is presented in section 6 , where an the adaptive controller is deduced. Finally, section 7 draws conclusions.

\section{HIV-1 infection model}

The model considered is the reduced complexity second order model

$$
\begin{gathered}
\dot{x}_{1}=s-d x_{1}-(1-u) \theta x_{1} x_{2} \\
\dot{x}_{2}=(1-u) \theta x_{1} x_{2}-\mu x_{2} .
\end{gathered}
$$

In equation (1), $s$ represents the production rate of healthy cells, the coefficient $d$ the natural death of the cells and $\theta$ the infection rate coefficient. The infection rate of healthy cells is proportional to the product of healthy cells $x_{1}$ and free virus $x_{3}$. This process can be influenced by drugs (Reverse Transcriptase Inhibitors - RTI) that reduce the virus ability to infect cells. This influence is represented by the manipulated variable $u$, in which $u=0$ corresponds to absence of drug and $u=1$ to a drug efficiency in preventing infection of $100 \%$. Actually, with the available drugs, the efficiency is below $100 \%$, and $u$ is constrained to the interval $\left[0, u_{\max }\right]$ with $u_{\max }<1$.

Equation (2) comprises two terms that represent, respectively, the transition of healthy cells to infected cells and the death of infected cells, with $\mu$ the death coefficient.

An infected cell liberates free virus. In this reduced complexity model the virus load is assumed to be proportional to the concentration of infected cells.

Table 1 shows one possible set of model parameters, used in simulations.

\begin{tabular}{cll}
\hline Parameter & Value & Units \\
\hline$d$ & 0.02 & day $^{-1}$ \\
$s$ & 10 & $\mathrm{~mm}^{-3}$ day $^{-1}$ \\
$\theta$ & $1 \times 10^{-3}$ & $\mathrm{~mm}^{3}$ day $^{-1}$ \\
$\mu$ & 0.24 & day $^{-1}$ \\
\hline
\end{tabular}

Table 1. Model parameters.

The reduced nonlinear model $(1,2)$, may also be written as

$$
\dot{x}=f(x)+g(x) u
$$


where the state vector is given by $x=\left[x_{1} x_{2}\right]^{\prime}$ and with the vector functions $f$ and $g$ defined as

$$
\begin{gathered}
f:=\left[\begin{array}{c}
s-d x_{1}-\theta x_{1} x_{2} \\
\theta x_{1} x_{2}-\mu x_{2}
\end{array}\right] \\
g:=\theta x_{1} x_{2}\left[\begin{array}{c}
1 \\
-1
\end{array}\right] .
\end{gathered}
$$

\section{Equilibrium points}

In the absence of therapy, i. e. when $u=0$, model $(1,2)$ has as equilibrium points the solutions of the algebraic equations

$$
\begin{aligned}
& 0=s-d x_{1}-(1-u) \theta x_{1} x_{2} \\
& 0=(1-u) \theta x_{1} x_{2}-\mu x_{2}
\end{aligned}
$$

with respect to the state variables $x_{1}$ and $x_{2}$. These equilibrium points are

$$
x_{1}=\frac{s}{d}, \quad x_{2}=0
$$

corresponding to an healthy person, and

$$
x_{1}=\frac{\mu}{\theta(1-u)}, \quad x_{2}=\frac{s}{\mu}-\frac{d}{\theta(1-u)}
$$

corresponding to an infected individual.

The local stability analysis of these equilibrium points is made by computing the eigenvalues of the Jacobian matrix $\widetilde{A}=\partial f / \partial x$, given by

$$
\widetilde{A}=\left[\begin{array}{cc}
-d-\theta x_{2} & -\theta x_{2} \\
\theta x_{2} & \theta x_{1}-\mu
\end{array}\right]_{x=x_{e q}}
$$

By using the model parameters of table 1, the results of table 2 are obtained.

\section{Exact Linearization}

System $(1,2)$ is not linearizable by performing a state transformation only. However, by the combined use of the transformations

$$
u=\alpha(x)+\beta(x) v
$$




\begin{tabular}{|rcc|}
\hline Equilibrium point: & {$\left[\begin{array}{cc}240.0000 & 21.6667\end{array}\right]^{T}$} \\
Eigenvalues: & $-0.0208 \pm 0.0690 j$ \\
Stability: & asymptotically stable \\
\hline Equilibrium point: & {$\left[\begin{array}{cc}500.0000 & 0.0000\end{array}\right]^{T}$} \\
Eigenvalues: & -0.0200, & 0.2600 \\
Stability: & unstable \\
\hline
\end{tabular}

Table 2. Stability of the equilibrium points of the reduced model.

$$
z=S(x)
$$

the following linear model is obtained

$$
\dot{z}=A z+B v
$$

with

$$
A=\left[\begin{array}{ll}
0 & 1 \\
0 & 0
\end{array}\right] \quad B=\left[\begin{array}{l}
0 \\
1
\end{array}\right] .
$$

The manipulated variable $v$ in the transformed model is called "virtual" because it has only mathematical existence, in opposition to $u$, that has the physical meaning of being the drug dosage actually applied to the patient. Equation (11) allows to compute the actual drug dose $u$ such that between $v$ and $z$ there is a linear relationship to which linear control techniques may then be applied.

Proposition 1 The transformations performing linearization are

$$
\begin{aligned}
& \beta(x)=\frac{1}{\theta x_{1} x_{2}(\mu-d)} \\
& \alpha(x)=\frac{-d s+d^{2} x_{1}+\mu^{2} x_{2}+(d-\mu) \theta x_{1} x_{2}}{(d-\mu) \theta x_{1} x_{2}} \\
& S(x)=\left[\begin{array}{c}
\varphi(x) \\
s-d x_{1}-\mu x_{2}
\end{array}\right]
\end{aligned}
$$

with $\varphi(x)$ given by

$$
\varphi(x)=x_{1}+x_{2}-\frac{\mu}{\theta}-\frac{s}{\mu}+\frac{d}{\theta} .
$$

Proof In [5] it is shown that the nonlinear system (3) with $f\left(x_{0}\right)=0$ and scalar input $u$ is feedback linearizable around the equilibrium $x_{0}$ if and only if the distributions $D_{i}$ defined by

$$
D_{i}=\operatorname{span}\left\{g(x), \operatorname{ad}_{f} g(x), \ldots, \operatorname{ad}_{f}^{i-1} g(x)\right\}
$$


verify the two following conditions:

$$
\begin{aligned}
& \operatorname{dim} D_{n}\left(x_{0}\right)=n, \\
& D_{n-1} \text { is involutive around } x_{0} .
\end{aligned}
$$

In relation to the model (3) with $f$ and $g$ given by (4) and (5), the first condition results in

$$
\begin{aligned}
\operatorname{dim} D_{2}(x) & =\operatorname{rank}[g(x) \\
& =\frac{\beta k}{c} x_{2} \operatorname{rank}\left[\begin{array}{cc}
x_{1} & s-\mu x_{1} \\
-x_{1} & -s+d x_{1}
\end{array}\right] \\
& =2, \text { for } x_{1}, x_{2} \neq 0 \text { and } \mu \neq d
\end{aligned}
$$

and hence $\operatorname{dim} D_{2}\left(x_{0}\right)=2$. The second condition is also verified because $D_{1}=$ $\operatorname{span}\{g(x)\}$ is involutive since the Lie bracket $[g, g]=0 \in D_{1}$. The model is therefore feedback linearizable.

Since the conditions on $D_{i}$ are satisfied, there exists ( [5]) a function $\varphi(x)$ that verifies the following three conditions:

$$
\begin{aligned}
& \varphi\left(x_{0}\right)=0 \\
& \left\langle\mathrm{~d} \varphi, a d_{f}^{k} g\right\rangle(x)=0, \quad k=0,1, \ldots, n-2 \\
& \left\langle\mathrm{~d} \varphi, a d_{f}^{n-1} g\right\rangle\left(x_{0}\right) \neq 0 .
\end{aligned}
$$

In terms of $\varphi(x)$, the linearizing transforms yielding (14) around the equilibrium state $x_{0}$ are given by [5]:

$$
\begin{aligned}
\alpha(x) & =-\left(L_{g} L_{f}^{n-1} \varphi(x)\right)^{-1} L_{f}^{n} \varphi(x) \\
\beta(x) & =\left(L_{g} L_{f}^{n-1} \varphi(x)\right)^{-1} \\
z_{i} & =L_{f}^{i-1} \varphi(x), \quad i=1,2, \ldots, n .
\end{aligned}
$$

The function 18 satisfies the three conditions, in particular

1. Computing $\varphi(x)$ at the equilibrium $x_{0}$ given by point (9) yields $\varphi\left(x_{0}\right)=0$;

2. $\langle\mathrm{d} \varphi, g\rangle=\frac{\partial \varphi(x)}{\partial x} g(x)=0$;

3. $\langle\mathrm{d} \varphi,[f, g]\rangle=\frac{\partial \varphi(x)}{\partial x}[f, g]=\frac{\beta k}{c}(d-\mu) x_{1} x_{2} \neq 0$, for $x=x_{0}$.

Using $\varphi(x)$ as given by (18) and (26)-(28) yields the transformations (15-17). 
The expression (18) for $\varphi(x)$ is obtained by noting that Condition 2 may be written as

$$
\left[\begin{array}{ll}
\frac{\partial \varphi}{\partial x_{1}} & \frac{\partial \varphi}{\partial x_{2}}
\end{array}\right]\left[\begin{array}{c}
1 \\
-1
\end{array}\right] \frac{\beta k}{c} x_{1} x_{2}=0
$$

and hence implies that $\varphi(x)$ satisfies the partial differential equation

$$
\frac{\partial \varphi}{\partial x_{1}}=\frac{\partial \varphi}{\partial x_{2}}
$$

whose solution is given by any differentiable function $\Phi$ of argument $x_{1}+x_{2}$ :

$$
\varphi\left(x_{1}, x_{2}\right)=\Phi\left(x_{1}+x_{2}\right) .
$$

The simplest choice that also satisfies Condition 1 is given by (18). The expressions for $\alpha$ and $\beta$ follow then in a straightforward way from (28).

With these transformations, the system in a region of state space around the equilibrium (9) is transformed exactly in the linear system (14).

\section{Control with known parameters}

The problem of designing a control law for the linearized system is addressed hereafter. The aim is to design a state feedback control law that generates the virtual manipulated variable $v$ as a function of the transformed state $z$. More specifically, we want to design the vector of feedback gains $K=\left[k_{1} k_{2}\right]$, the equilibrium value of $v$ (denoted $\bar{v}$ ) and the equilibrium $\bar{z}=\left[\bar{z}_{1} \bar{z}_{2}\right]^{T}$ of $z$ corresponding to the a specified equilibrium of $x$, in the control law:

$$
v=\bar{v}-K \widetilde{z}
$$

where

$$
\widetilde{z}:=z-\bar{z}
$$

The equilibrium value of the control variable of the linear system verifies

$$
A \bar{z}+B \bar{v}=0 .
$$

\subsection{Equilibrium values}

Assume that the concentration of infected cells $x_{2}$ is to be driven to a reference value $r$ and kept there. At the equilibrium defined by $x_{2}=r$ one has, by equating the derivatives to zero in $(1,2)$

$$
u=1-\frac{\mu d}{\theta(s-\mu r)}
$$


and

$$
x_{1}=\frac{s-\mu r}{d} .
$$

In terms of the linearized system (that operates with transformed variables) this results in the equilibrium point $\bar{z}=S(\bar{x})$, i. e.:

$$
\left[\begin{array}{l}
\bar{z}_{1} \\
\bar{z}_{2}
\end{array}\right]=\left[\begin{array}{c}
\frac{s-\mu r}{d}+r+\frac{1}{\theta}(d-\mu)-\frac{s}{\mu} \\
0
\end{array}\right]=T(r) .
$$

\subsection{LQ controller design}

It is then possible to design a LQ controller, using the linearized dynamics, that keeps the system at the desired reference value $r$.

The transformation $T(r)$ allows to compute the equilibrium point in terms of the variables $\left(z_{1}, z_{2}\right)$. The controller is then designed by minimizing the quadratic cost

$$
J=\int_{0}^{+\infty} z^{T} Q_{z} z+\rho v^{2} \mathrm{~d} t
$$

where $Q_{z}$ and $\rho$ adjust the contribution of the variables $z(t)$ and $v(t)$. Since these variables are virtual (corresponding to transformed states) it is difficult to develop heuristic choices of their values. Thus, it was decided to adjust the weights $Q_{x}$ for the state variables $x$ and then compute the corresponding $Q_{z}$. Using a linear approximation, it is concluded that

$$
Q_{z}=\left(\frac{\partial S^{-1}}{\partial z}\right)^{T} Q_{x}\left(\frac{\partial S^{-1}}{\partial z}\right)
$$

These weights are "tuning knobs" that allow the designer to adjust the relative importance of the state variables and the drug usage.

\section{Adaptive Control}

In practice model parameters are not perfectly known. A possible approach to estimate them is described hereafter and relies on a joint control Lyapunov function for both the control and estimation errors [7]. In the work reported only the adaptation of the infection parameter $\theta$ is considered.

\subsection{Error equation}

Let $\theta^{*}$ be the (unknown) true value of parameter $\theta$, assumed to be constant, and $\hat{\theta}$ its estimate. The estimation error $\widetilde{\theta}$ verifies

$$
\hat{\theta}=\theta^{*}+\widetilde{\theta}
$$


Differentiating (12) with respect to time and using the change of variable (11) yields

$$
\dot{z}=\frac{\partial S}{\partial x}\left\{f\left(x, \theta^{*}\right)+g\left(x, \theta^{*}\right)[\alpha(x, \hat{\theta})+\beta(x, \hat{\theta}) v]\right\} .
$$

For the adaptive technique to be applied the equation error that relates the tracking error with the estimation error should be linear in $\widetilde{\theta}$. For this sake, we do a first order expansion of both $\alpha$ and $\beta$ and neglect higher order terms:

$$
\begin{aligned}
& \alpha\left(x, \theta^{*}\right) \approx \alpha(x, \hat{\theta})-\widetilde{\alpha}(x, \hat{\theta}) \widetilde{\theta} \\
& \beta\left(x, \theta^{*}\right) \approx \beta(x, \hat{\theta})-\widetilde{\beta}(x, \hat{\theta}) \widetilde{\theta}
\end{aligned}
$$

where

$$
\widetilde{\alpha}(x, \hat{\theta})=\frac{\partial \alpha}{\partial \hat{\theta}}=\frac{d s-d^{2} x_{1}-\mu^{2} x_{2}}{(d-\mu) x_{1} x_{2} \hat{\theta}^{2}}
$$

and

$$
\widetilde{\beta}(x, \hat{\theta})=\frac{\partial \beta}{\partial \hat{\theta}}=\frac{1}{\hat{\theta}^{2}(d-\mu) x_{1} x_{2}} .
$$

With this approximation, and using the fact that

$$
\frac{\partial S}{\partial x}\left\{f\left(x, \theta^{*}\right)+g\left(x, \theta^{*}\right)\left[\alpha\left(x, \theta^{*}\right)+\beta\left(x, \theta^{*}\right) v\right]\right\}=A z+B v
$$

equation (41) becomes

$$
\dot{z}=A z+B v+\Psi(x, v, \theta) \widetilde{\theta}
$$

where

$$
\Psi(x, v, \theta):=-\frac{\partial S}{\partial x} g(x, \hat{\theta})[\widetilde{\alpha}(x, \hat{\theta})+\widetilde{\beta}(x, \hat{\theta}) v] .
$$

From this equation and using (32) and (34) the error equation is written as

$$
\dot{\tilde{z}}=A_{k} \widetilde{z}+\Psi(x, v, \theta) \widetilde{\theta} .
$$

Since

$$
\frac{\partial S}{\partial x}=\left[\begin{array}{cc}
1 & 1 \\
-d & -\mu
\end{array}\right]
$$

it follows that

$$
\Psi(x, v, \hat{\theta})=\left[\begin{array}{l}
0 \\
1
\end{array}\right] \frac{v+d s-d^{2} x_{1}^{2}-\mu^{2} x_{2}}{\hat{\theta}} .
$$




\subsection{Adaptation law}

In order to design an adaptation mechanism to adjust $\theta$, consider the candidate Control Lyapunov Function

$$
V(\widetilde{z}, \widetilde{\theta})=\widetilde{z}^{T} P \widetilde{z}+\frac{1}{\gamma} \widetilde{\theta}^{2}
$$

where $P=P^{T}$ is a positive definite matrix and $\gamma>0$ is a scalar design parameter. Differentiating $V$ with respect to time and using (49) it follows that

$$
\dot{V}=\widetilde{z}^{T}\left(A_{k}^{T} P+P A_{k}\right) \widetilde{z}+\widetilde{\theta}\left(2 \Psi^{T}(x, v, \hat{\theta}) P \widetilde{z}+\frac{2}{\gamma} \dot{\tilde{\theta}}\right)
$$

where

$$
A_{k}:=A-B K .
$$

For $K$ such that $A_{k}$ is hurwitz (as it happens, for instance, if $K$ is designed by solving the LQ problem in section 5.1), there is $Q=Q^{T}$ positive definite such that

$$
A_{k}^{T} P+P A_{k}=-Q .
$$

Using (55) and selecting $\dot{\widetilde{\theta}}$ such that

$$
\dot{\tilde{\theta}}=-\gamma \Psi(x, v, \hat{\theta})^{P} \widetilde{z}
$$

equation (53) becomes

$$
\dot{V}=-\widetilde{z}^{T} Q \widetilde{z} \leqslant 0 \quad \forall \widetilde{z} \neq 0 .
$$

Using a standard argument based on La Salle's Invariant Set Theorem it is then concluded that $\widetilde{z} \rightarrow 0$. Equation (56) implies the use of an adaptation law given by

$$
\hat{\boldsymbol{\theta}}(t)=-\hat{\boldsymbol{\theta}}(0)+\int_{0}^{t} \gamma \Psi^{T}(x, v, \hat{\boldsymbol{\theta}}) P \widetilde{z} d t
$$

or

$$
\hat{\theta}(t)=\hat{\theta}(0)+\gamma \int_{0}^{t} \frac{d^{2} x_{1}+\mu^{2} x_{2}-d s-v}{\hat{\theta}}\left(p_{12} \widetilde{z}_{1}+p_{22} \widetilde{z}_{2}\right) d t .
$$

Figure 1 shows a result obtained using adaptive controller.

\section{Conclusions}

It was shown that a reduced complexity nonlinear model for the HIV-1 infection can be controlled using adaptive nonlinear control methods. The approach followed combines exact linearization, LQ control and a joint control Lyapunov function for for the 

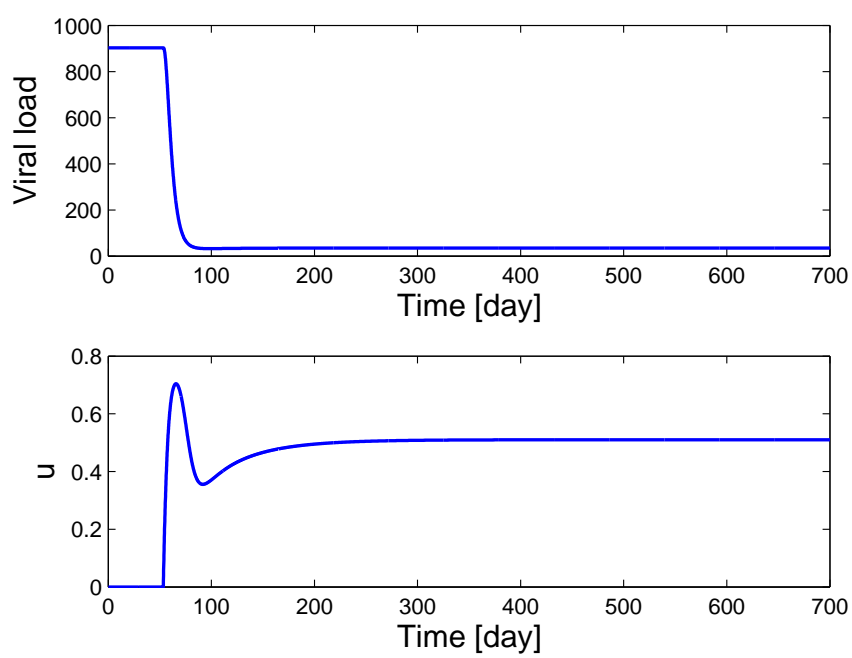

Figure 1. Results with adaptive control.

tracking and estimation errors, to design the estimation law. Since this requires a linear dependence of the error equation on the estimation error, some approximations related to the linearizing transforms have to be performed.

The adaptation law considers only the tuning of the infection parameter. The same procedure may be extended to estimate the other parameters at the cost of a more cumbersome algebra.

\section{References}

[1] F.L. BIAfORE and C.E. D' ATELlis: Exact linearization and control of a HIV-1 predator-prey model. Proc. 2005 IEEE Eng. in Medicine and Biology 27th Annual Conf., Shanghai, China, (2005), 2367-2370.

[2] M.E. BRANDT and G. CHEN: Feedback control of a bioidynamical model of HIV1. IEEE Trans. Biomedical Eng., 48(7), (2001), 754-759.

[3] C.-F. Cheng and C.-T. Chang: Viral load analysis of a biodynamical model of HIV-1 with unknown equilibrium points. Proc. IEEE Conf. on Control Applications, Taipei, Taiwan, (2004), 557-561.

[4] S. GeE, Z. TIAN and T. LEE: Nonlinear control of a dynamic model of HIV-1. IEEE Tran. Biomedical Eng., 52(3), (2005), 353-361. 
[5] H. NiJMeijer and A. VAN DER Schaft: Nonlinear Dynamical Control Systems. Springer-Verlag, 1990.

[6] A. Perelson and P. Nelson: Mathematical analysis of HIV-1 dynamics in vivo. SIAM Review, 41(1), (1999), 3-44.

[7] S. SASTRY and A. ISIDORI: Adaptive control of linearizable systems. IEEE Trans. on Automatic Control, 34(11). (1989), 1123-1131.

[8] J.F. De Souza, M. Caetano and T. Yoneyama: Optimal control applied to the antiviral treatment of AIDS. Proc. 39th IEEE Conf. on Decision and Control, Sydney, Australia, (2000), 4839-4844.

[9] Z. TIAN, S.S. GE and T.H. LEE: Globally stable nonlinear control of HIV-1 systems. Proc. Amer. Control Conf., Boston, Massachusets, (2004).

[10] R. ZuRAKOWSKi and A. TeEL: A model predictive control based scheduling method for HIV therapy. J. of Theoretical Biology, 238 (2006), 368-382. 